\title{
STAR FORMATION ACTIVITY IN HIGH $\mathbf{M}_{H} / \mathrm{L}_{B}$ GALAXIES
}

\author{
L. VAN ZEE \\ National Radio Astronomy Observatory \\ PO Box O, Socorro, NM 87801, USA
}

\begin{abstract}
The results of an investigation of the evolutionary status of fifteen gas-rich, low surface brightness dwarf galaxies (LSBDGs) are presented. LSBDGs are defined by unusually high values of $\mathrm{M}_{H} / \mathrm{L}_{B}$ and the presence of extended $\mathrm{HI}$ envelopes. At both the present epoch and the past, the star formation process appears to be inefficient: the LSBDGs are underluminous for their HI mass and very little active star formation is currently observed. Analysis of global optical colors and elemental enrichments indicate that these objects are not "young" systems; rather, star formation has been occurring for several Gyr. One clue to the star formation history of these objects is that the global gas density is significantly lower than the Toomre instability criterion throughout the gas disk. Local peaks in the HI surface density, however, approach the instability criterion and are correlated with sites of active star formation. Thus, while star formation appears to be inhibited globally, the local gas distribution plays a crucial role in regulating the star formation activity in these low mass galaxies.
\end{abstract}

\section{Introduction}

As part of an investigation of the onset of star formation in low mass galaxies, we have undertaken a study of the evolutionary status of fifteen isolated, gas-rich, low surface brightness dwarf galaxies (LSBDGs) which appear to have inefficient star formation processes. The LSBDGs are distinguished by unusually high values of $\mathrm{M}_{H} / \mathrm{L}_{B}$ and the presence of extended HI envelopes (van Zee et al. 1995). Both of these physical properties suggest that the star formation process is inefficient in the LSBDGs since they have not been able to convert their abundant supply of gas into stars. One possibility is that these galaxies are still in the process of collapse and thus have not yet processed the majority of their gas. Alternatively, the star formation process may be inefficient because the global gas density falls below the critical density for star formation.

To address these possibilities, we have examined the gas distribution and constrained the evolutionary status of the dominant stellar populations of a sample of LSBDGs. High spatial and spectral resolution HI synthesis observations were undertaken of 6 LSBDGs with the VLA in order to investigate the global and local gas surface densities (van Zee et al. 1997a). Deep optical images of 15 LSBDGs were obtained with the KPNO $0.9 \mathrm{~m}$; optical colors place constraints on the age of the dominant stellar population (Bruzual \& Charlot 1993).

\section{Results and Discussion}

Based on UBVR colors, the LSBDGs have undergone quasi-continuous star formation for at least the last few Gyr, yet their current star formation rates are extremely low (van Zee et al. 1997b). These galaxies are not "young" systems. Rather, star formation has been underway for a significant period of time, but the process of converting gas into stars appears to be inefficient.

Analysis of the HI synthesis images reveals that the majority of the HI gas appears to be below the density threshold required for star formation (van Zee et al. 1997a). Thus, the star formation process appears to be inhibited in these objects because the local gas surface density rarely crosses the critical density threshold. Similar studies of a small sample of "normal" dwarf galaxies, however, suggest that the HI gas in these systems is also well below the critical threshold. 
In fact, it is impossible to distinguish between these two types of galaxies based on HI content alone.

These results suggest that the local gas density plays a critical role in regulating star formation activity. Star formation may be inhibited in dwarf galaxies because the local gas density rarely crosses the gravitational instability threshold. However, it is also possible that star formation is inefficient in the LSBDGs simply because massive star formation has never been induced via an interaction. In the future, it will be beneficial to investigate the star formation histories and gas distributions of a large sample of apparently isolated dwarf galaxies which do have efficient star formation processes in order to distinguish between these mechanisms.

\section{References}

Bruzual, G., \& Charlot, S. 1993, ApJ, 96, 877

van Zee, L., Haynes, M.P., Giovanelli, R. 1995, AJ, 109, 990

van Zee, L., Haynes, M.P., Salzer, J.J., Broeils, A.H. 1997a, AJ, 113, 1618

van Zee, L., Haynes, M.P., Salzer, J.J. 1997b, AJ, in press 